\title{
Learning-by-Doing, Produtividade Agrícola e Crescimento Econômico
}

\author{
Rosemeiry Melo Carvalho*, Flávio Ataliba F. D. Barreto ${ }^{\dagger}$
}

Sumário:1. Introdução; 2. Descrição do Modelo; 3. Determinação do Nível de Emprego; 4. Relação entre Produtividade Agrícola e Bem-Estar; 5. Conclusões;

Palavras-chave: Learning-by-Doing; Produtividade Agrícola; Bem-Estar.

Códigos JEL: Q17; 033; O47.

Este artigo procura explicar a influência da produtividade agrícola sobre o emprego, renda e bem-estar de economias abertas utilizando uma extensão ao modelo do Matsuyama (1992). O modelo originalmente proposto difere do modelo aqui apresentado em relação a duas suposições básicas. Primeiro considera-se que a dinâmica da economia está condicionada a evolução da produtividade nos setores agrícola e industrial. Adicionalmente, supõe-se que não há spillovers entre as economias. A partir dos resultados obtidos pode-se verificar que as conclusões em Matsuyama representam um caso particular de conclusões mais gerais, onde dependendo da magnitude dos parâmetros observados, os ganhos de produtividade na agricultura podem não reduzir o crescimento da economia, de forma que não existe necessariamente uma relação negativa entre produtividade agrícola e crescimento econômico, como caracterizado no modelo de Matsuyama (1992). Por outro lado, o fato de uma economia se especializar na agricultura não necessariamente implicaria em perda de bem-estar tanto no curto quanto no longo prazo.

This paper tries to explain the influence of agricultural productivity on employment, income and welfare of open economies. Using an extension of Matsuyama (1992) model, the paper proves that does not exist necessarily an inverse relation between growth and agricultural productivity. Crucial to this result are the assumptions that the dynamic of the economy is not solely based on industrial productivity, but it relies also on agricultural productivity; and that there

* Professora do Departamento de Economia Agrícola - DEA UFC. Universidade Federal do Ceará. Departamento de Economia Agrícola. Caixa Postal 6017-Pici. CEP 60451-970. Fortaleza-Ceará. rmelo@ufc.br

†Professor do Curso de Pós-Graduação em Economia - CAEN UFC. Universidade Federal do Ceará. Curso de Pós-Gradução em Economia - CAEN. Av. da Universidade, 2700. $2^{\circ}$. Andar. CEP 60451-970. Fortaleza-Ceará. ataliba@ufc.br. 
are spillovers among economies. Under this perspective Matsuyama's result is a particular case of this more general approach. On the other hand, specialization in agriculture does not lead inexorably to welfare losses either in the short or in the long run.

\section{INTRODUÇÃO}

A importância da tecnologia e do conhecimento no processo de crescimento econômico tem sido reforçada nos últimos anos com o aprofundamento das relações comerciais entre os países. Esse movimento vem revigorando o debate, iniciado principalmente pela CEPAL (Comissão Econômica para América Latina) na década de 1950, sobre o padrão de especialização que as economias devem assumir especialmente no que se refere ao desempenho dos países mais pobres em um cenário mundial de maior integração comercial.

A partir de meados da década de 1980, especialmente com o surgimento do que se convencionou chamar de " modelos de crescimento endógenos", uma forte luz tem se colocado no melhor entendimento das relações entre o comércio e o crescimento da renda per capita dos países no longo prazo. Para Lucas (1988), se as vantagens comparativas de um país em um determinado segmento forem reforçadas por um processo acelerado de learning-by-doing, eles tenderão a divergir daquelas economias que se especializam em segmentos com baixo processo de aprendizado.

Grossman e Helpman (1989, 1990, 1991a,b), Romer (1990), Krugman (1990) e Rivera-Batiz e Romer (1991a,b), tem chamado a atenção para o fato de que o livre comércio pode levar a um padrão de crescimento mais acelerado através do surgimento das economias de escala e dos chamados spillovers tecnológicos. Nessa direção, Young (1991) e Stokey (1991) sugerem que, como o processo de inovação e spillover do conhecimento ocorrem com maior velocidade entre as economias mais industrializadas, o padrão de crescimento desses países tende a ocorrer de forma mais acelerada que nos países não industrializados. De acordo com Quad (1997), a formação de clubes de convergências entre países de alta e baixa renda per capita nos últimos 30 anos é uma importante evidência desse processo.

Uma importante contribuição a esse debate teórico foi apresentada por Matsuyama (1992), onde pressupõem-se a existência de duas economias, uma doméstica e outra caracterizada como o resto do mundo. Em cada uma delas a atividade produtiva está dividida em dois setores, um industrial e outro agrícola. No primeiro, assume-se a existência de certa dinâmica através de um processo de learning-bydoing, enquanto que o segundo é caracterizado pela impossibilidade de crescimento de sua produtividade ao longo do tempo.

Sob essas suposições, e considerando um ambiente de livre comércio, a economia que apresentar vantagens comparativas iniciais no setor industrial crescerá ao longo do tempo enquanto que a economia que se especializar na agricultura irá se estagnar. Ademais, considerando que os setores industrial e agrícola competem por mão-de-obra, um choque de produtividade na agricultura reduziria o emprego na indústria, diminuindo sua produção e , conseqüentemente, a taxa de crescimento. Esse efeito estabeleceria a existência de uma relação inversa entre produtividade agrícola e o crescimento econômico. Evidentemente, como essas conclusões estão substanciadas pela inexistência de algum processo que gere ganhos tecnológicos também no setor agrícola, economias industrializadas e agrícolas irão necessariamente apresentar taxas de crescimento diferenciadas ao longo do tempo.

No entanto, diversos estudos têm evidenciado nas últimas décadas o crescimento da produtividade do setor agrícola em diversos países. Kawagoe et alii (1985), Lau e Yotopoulos (1989), Bureau et alii (1995) documentaram um expressivo crescimento da produtividade agrícola para diversos países entre 1960 e 1980. Essa tendência é corroborada também para anos mais recentes como pode ser visto em Fulginiti e Perrin (1993), Battese e Rao (2001) e Battese et alii (2001).

Em um importante estudo Prasadasa e Coelli (1998) analisaram o crescimento da produtividade da agricultura em 96 países entre 1980 e 1995, considerando na amostra 27 países da África, 11 da América 
do Norte e Central, 10 da América do Sul, 23 da Ásia, 22 da Europa, 3 da Austrália e os países da antiga USSR. Os resultados obtidos mostraram um aumento médio anual de $2,7 \%$ na produtividade total dos fatores na agricultura sendo mais expressivo os resultados obtidos na Ásia $(3,1 \%)$.

No Brasil, Barros (1999) estimou que, a produtividade agrícola brasileira cresceu a uma taxa média de 3,6\% ao ano, entre 1975 e 1995 sendo que a produtividade da terra apresentou um crescimento anual de $2,47 \%$ enquanto que a do trabalho foi de $3,26 \%$. Vicente et alii (2001) calcularam que os ganhos totais de produtividade nesse período seriam na ordem de $95 \%$ e mais recentemente, usando método de fronteira estocástica, Marinho e Carvalho (2004) verificaram que esses ganhos situavam-se na ordem de $41 \%$, considerando o mesmo período.

Deste modo, diante de tais evidências, tornam-se pouco realista estudos teóricos que negligenciam a influência da produtividade da agricultura no processo de crescimento da renda per capita em economias sujeitas ao livro comércio. Tomando por base o trabalho de Matsuyama (1992), este artigo procura verificar quais são os efeitos sobre o nível de emprego, produto e bem-estar quando se introduz a possibilidade de ganhos de produtividade também no setor agrícola. Adicionalmente, este artigo admite a possibilidade de que os parâmetros de produtividade dos setores entre as economias sejam distintos, como é mais razoável supor. Tais suposições podem ajudar a explicar a especialização de determinadas economias em setores distintos ao padrão inicial de suas vantagens comparativas. Por outro lado podese mostrar que a especialização na agricultura não necessariamente levaria a um menor crescimento da renda per capita ou perca de bem-estar em uma economia.

Além dessa seção introdutória são apresentadas mais cinco seções. Na segunda faz-se a descrição do modelo para a economia doméstica e o resto do mundo. A terceira seção analisa o padrão inicial e a dinâmica do emprego na economia doméstica enquanto a quarta é dedicada à análise de bem-estar. Por fim, na última seção, são apresentadas algumas principais conclusões desse artigo.

\section{DESCRIÇÃO DO MODELO}

Assim como em Matsuyama (1992), este modelo é essencialmente uma variação da estrutura teórica de Ricardo-Viner-Jones (Wong (1995)), seguindo as seguintes hipóteses iniciais:

I Existe uma quantidade contínua de economias abertas, as quais nãoo são suficientemente grandes para influenciar o comportamento das demais. Uma delas é chamada economia doméstica e as demais de resto do mundo;

II Cada economia é composta por empresas e famílias;

III A dotação de fatores em cada economia é fixa e os bens são produzidos em dois setores distintos, industrial e agrícola;

IV Utiliza-se trê insumos básicos: capital industrial, capital agrícola e mão-de-obra;

V O capital industrial e o capital agrícola são de uso exclusivo em cada setor;

VI A mão-de-obra é considerada homogÊnea e pode se deslocar entre os setores, mas não entre as economias;

VII Toda a mão-de-obra disponével está plenamente empregada e não existem diferenças salariais entre os setores;

VIII As tecnologias de produção são homogêneas de grau um e exibem retornos decrescentes nos dois setores;

IX As produtividades aumentam ao longo do tempo através do processo de learning-by-doing; 
$\mathrm{X}$ Não existem spillovers de conhecimento entre os setores nem entre as economias.

As hipóteses apresentadas referem-se a uma estrutura teórica onde as economias, doméstica e resto do mundo, atuam em um ambiente perfeitamente competitivo, ou seja, os preços dos fatores de produção e dos produtos são dados tanto para as empresas quanto para as famílias.

Para isolar os efeitos da evolução da produtividade agrícola e industrial considera-se que em cada economia cada um dos setores possui dotações fixas de capital ao longo do tempo. Desse modo as alterações nos níveis de produção dependerão apenas do deslocamento da mão-de-obra entre os setores, a qual é homogênea e a condição de competição perfeita assumida anteriormente, assegura que não haja diferenças salariais entre os setores.

A existência de fatores fixos na função de produção determina retornos decrescentes de escala e para que as funções possam ser apresentadas na sua forma intensiva assume-se homogeneidade de grau 1.

As hipóteses referentes ao aumento de produtividade pelo processo de learning-by-doing e a não existência de spillovers de conhecimento permitem que se análise os efeitos das variações da produtividade em cada setor no produto da economia e no nível de bem-estar de forma isolada.

\subsection{A Economia Doméstica}

\subsubsection{Tecnologia de produção da indústria}

Considere a tecnologia de produção da indústria descrita pela função de produção:

$$
Y_{I t}=I_{t} F\left(L_{I t}, K\right) \quad F(0)=0, F^{\prime}>0, F^{\prime \prime}<0
$$

onde

- $Y_{I t}$ é a quantidade produzida de bens industrializados no período $t$;

- $I_{t}$ corresponde à produtividade do setor industrial;

- $L_{I t}$ representa a parcela da mão-de-obra empregada nesse setor;

- $K$ é a quantidade de capital utilizada na indústria.

Como a mão-de-obra está plenamente empregada nos setores industrial e agrícola, tem-se:

$$
L_{I t}+L_{A t}=1
$$

onde $L_{A t}$ representa a parcela da mão-de-obra empregada no setor agrícola e a quantidade total de trabalho ofertada $(L)$ é normalizada por um.

Dividindo a equação 1 pela quantidade utilizada de capital nesse setor, tem-se:

$$
\frac{Y_{I t}}{K}=I_{t} F\left(\frac{L_{I t}}{K}, \frac{K}{K}\right)
$$

ou ainda,

$$
y_{I t}=I_{t} F\left(\frac{L_{I t}}{K}, 1\right)
$$

Fazendo $y_{I t} \equiv Y_{I t} / K$ e $l_{I t} \equiv L_{I t} / K$ em (3a), a produção do setor industrial por unidade utilizada de capital pode ser representada na sua forma intensiva como:

$$
y_{I t}=I_{t} f\left(l_{I t}\right)
$$




\subsubsection{Tecnologia de produção da agricultura}

A tecnologia de produção do setor agrícola é dada por:

$$
Y_{A t}=A_{t} G\left(L_{A t}, T\right) \quad G(0)=0, G^{\prime}>0, G^{\prime \prime}<0
$$

onde

- $Y_{A t}$ é a quantidade produzida de bens agrícolas no período $t$, excluindo-se a parcela destinada ao consumo de subsistência, considerando-se apenas a produção destinada à comercialização. Assim tem-se que, $Y_{A t}>\gamma L$, onde $\gamma$ representa o nível de consumo de subsistência; ${ }^{1}$

- $A_{t}$ corresponde à produtividade do setor agrícola;

- $L_{A t}$ representa a parcela da mão-de-obra empregada nesse setor;

- $T$ é a quantidade de capital utilizada na agricultura.

De modo semelhante ao produto industrial, a produção agrícola pode ser representada na sua forma intensiva como:

$$
y_{A t}=A_{t} f\left(l_{A t}\right)
$$

onde $y_{A t} \equiv Y_{A t} / T$ e $l_{A t} \equiv L_{A t} / T$.

Dado que os estoques de capital industrial e agrícola são fixos, existe uma relação negativa entre as participações relativas da mão-de-obra nesses setores, ou seja, ${ }^{2}$

$$
l_{I t}=j\left(l_{A t}\right) \quad j^{\prime}<0
$$

\subsubsection{Evolução da produtividade}

Supõe-se que o nível tecnológico nos dois setores evolui ao longo do tempo devido a um processo de learning-by-doing. Os efeitos da experiência prática são puramente externos às firmas que os geram. Com completo Spillovers, as decisões de produção e emprego das firmas são feitas tomando como dados seus níveis de produtividade.

As produtividades industrial e agrícola evoluem ao longo do tempo de acordo com as equações:

$$
\begin{array}{cc}
\dot{I}_{t}=\delta Y_{I t} & \delta>0 \\
\dot{A}_{t}=\nu Y_{A t} & \nu>0
\end{array}
$$

Em Matsuyama (1992), o processo de learning-by-doing afeta apenas a evolução da produtividade no setor industrial. Neste modelo considera-se também que há ganhos de produtividade na agricultura, de modo que a dinâmica da economia está condicionada a evolução da produtividade nos dois setores.

\footnotetext{
${ }^{1} \mathrm{O}$ parâmetro $\gamma$ satisfaz à condição $A G(1)>\gamma L>0$. Deste modo, a quantidade de bens produzida no setor agrícola é mais que suficiente para garantir o nível de consumo de subsistência.

${ }^{2}$ Considerando a hipótese de pleno emprego, toda a força de trabalho da economia $(L)$ está distribuída entre os setores industrial e agrícola, $L^{I}+L^{A}=L$. Assim, tem-se que $K l_{I t}+T l_{A t}=1 \Rightarrow l_{I t}=1-\frac{T}{K} l_{A t}$. Então, $l_{I t}=j\left(l_{A t}\right)$.
} 


\subsubsection{Equilíbrio no mercado de trabalho}

A partir das equações 4 e 6, pode-se obter as produtividades marginais da mão-de-obra nos dois setores:

$$
\begin{gathered}
\frac{d y_{I t}}{d l_{I t}}=I_{t} f^{\prime}\left(l_{I t}\right) \\
\frac{d y_{A t}}{d l_{A t}}=A_{t} g^{\prime}\left(l_{A t}\right)
\end{gathered}
$$

A competição por mão-de-obra entre os dois setores estabelece que os valores das produtividades marginais desse fator sejam iguais, conduzindo à seguinte condição de equilíbrio no mercado de trabalho:

$$
A_{t} g^{\prime}\left(l_{A t}\right)=p_{t} I_{t} f^{\prime}\left(l_{I t}\right)
$$

onde $p_{t}$ representa a relação entre os preços dos bens industrializados e agrícolas, ou seja, $p_{t} \equiv$ $p_{\text {It }} / p_{\text {At }}$.

\subsubsection{Preferência dos Consumidores}

Considera-se que os indivíduos nas duas economias consomem bens agrícolas e industrializados e que suas preferências sejam idênticas e não-homotéticas ${ }^{3}$ de modo que eles maximizam uma função utilidade intertemporal dada por:

$$
U=\int_{0}^{\infty} u\left(c_{A t}, c_{I t}\right) e^{-\rho t} d t
$$

onde $c_{A t}$ e $c_{I t}$ referem-se aos consumos per capita de bens agrícolas e bens industrializados, e $p$ é a taxa de preferência dos indivíduos.

A presença de bens agrícolas na função utilidade implica a necessidade de atendimento da Lei de Engel, onde especifica que a elasticidade-renda por esses tipos de bens deva ser menor que a unidade, a partir de um determinado nível de renda ou consumo. Desta forma, é oportuna a especificação de preferências do tipo Stone-Geary de modo que somente o consumo de bens agrícolas além do nível de subsistência $\gamma$, possa gerar bem-estar, ou seja, a função de utilidade instantânea passa a ser definida apenas para $c_{A t} \geq \gamma$.

Como pode ser visto em Sharif (1986) e World (1990), a idéia de se utilizar um nível mínimo de consumo de bens agrícolas baseia-se na necessidade de um indivíduo obter uma quantidade mínima de calorias para garantir sua sobrevivência mesmo em sociedades não industrializadas. Desta forma, considerando essas necessidades, a função utilidade instantânea pode ser representada por:

$$
u\left(c_{A t}, c_{I t}\right)= \begin{cases}\frac{\left[\left(c_{A t}-\gamma\right)^{\beta} c_{I t}\right]^{(1-\sigma)}-1}{(1-\sigma)} & \text { para } \sigma>0, \sigma \neq 1 \\ \beta \log \left(c_{A t}-\gamma\right)+\log \left(c_{I t}\right) & \text { para } \sigma=1\end{cases}
$$

onde $\sigma$ é a elasticidade de substituição entre o consumo de bens agrícolas e industrializados.

As preferências Stone-Geary (PSG) tem sido amplamente utilizada na recente literatura de crescimento econômico. Rebelo (1992) aponta que, uma ampla classe de modelos de crescimento endógenos

\footnotetext{
${ }^{3}$ Uma relação de preferência monotônica em $x=\Re_{+}^{L}$ é homotética se todos os conjuntos de indiferença estão relacionados por uma expansão ao longo do raio; isto é, se $x \sim y$, então $\alpha x \sim \alpha y$ para qualquer $\alpha \geq 0$. No entanto, considerando-se que os bens agrícolas apresentam um nível de consumo de subsistência positivo, ou seja, $\gamma>0$, a função utilidade torna-se não-homotética, de modo que todos os conjuntos de indiferença estão dispostos paralelamente ao longo do eixo que representa o consumo desse bem.
} 
é inconsistente com a diversidade na taxa de crescimento entre países diante do mercado de capitais internacional. Uma das soluções para esse problema teórico é a utilização de um modelo de crescimento linear com preferências Stone-Geary. Easterly (1991) e Ben-David (1994) usam também PSG para construir modelos de crescimento que apresentam a possibilidade de diferentes taxas de equilíbrio de longo prazo. Mais recentemente Steger (2000) evidencia que grandes divergências nas taxas de crescimento entre países podem ser parcialmente explicados pela idéia de consumo de subsistência.

\subsection{A Economia Mundial}

\subsubsection{Tecnologia de produção da indústria}

A tecnologia de produção da indústria no resto do mundo é dada por:

$$
Y_{I t}^{*}=I_{t}^{*} F\left(L_{I t}^{*}, K^{*}\right) \quad F(0)=0, F^{\prime}>0, F^{\prime \prime}<0
$$

onde:

- $Y_{I t}^{*}$ é a quantidade produzida de bens industrializados no período $t$;

- $I_{t}^{*}$ refere-se à produtividade do setor industrial;

- $L_{I t}^{*}$ representa a parcela da mão-de-obra empregada nesse setor;

- $K^{*}$ é a quantidade de capital utilizada na indústria.

Considerando-se também que a mão-de-obra està plenamente empregada nos setores industrial e agrícola, normalizando a quantidade total de trabalho ofertada $(L)$ para 1 e dividindo 15 pela quantidade utilizada de capital $\left(K^{*}\right)$, tem-se:

$$
y_{I t}^{*}=I_{t}^{*} f\left(l_{I t}^{*}\right)
$$

onde $y_{I t}^{*} \equiv Y_{I t}^{*} / K^{*}$ e $l_{I t}^{*} \equiv L_{I t}^{*} / K^{*}$.

\subsubsection{Tecnologia de produção na agricultura}

A função de produção do setor agrícola do resto do mundo é dada por:

$$
Y_{A t}^{*}=A_{t}^{*} G\left(L_{A t}^{*}, T^{*}\right) \quad G(0)=0, G^{\prime}>0, G^{\prime \prime}<0
$$

onde:

- $Y_{A t}^{*}$ é a quantidade produzida de bens agrícolas no período t, excluindo-se a parcela destinada ao consumo de subsistência;

- $A_{t}^{*}$ corresponde à produtividade do setor agrícola;

- $L_{A t}^{*}$ representa a parcela da mão-de-obra empregada nesse setor;

- $T^{*}$ é a quantidade de capital utilizada na agricultura.

Em termos de unidade de capital, a produção agrícola pode ser representada por:

onde $y_{A t}^{*} \equiv Y_{A t}^{*} / T^{*}$ e $l_{A t}^{*} \equiv L_{A t}^{*} / T^{*}$.

$$
y_{A t}^{*}=A_{t}^{*} f\left(l_{A t}^{*}\right)
$$

No resto do mundo os estoques de capital industrial e agrícola também são fixos, existindo uma relação negativa entre as participações relativas da mão-de-obra nos dois setores.

$$
l_{I t}^{*}=j^{*}\left(l_{A t}^{*}\right) \quad\left(j^{*}\right)^{\prime}<0
$$




\subsubsection{Evolução da produtividade}

Assim como na economia doméstica, os níveis de produtividade industrial e agrícola evoluem devido a um processo de learning-by-doing de acordo com as expressões:

$$
\begin{array}{ll}
\dot{I}_{t}^{*}=\delta^{*} Y_{I t}^{*} & \delta^{*}>0 \\
\dot{A}_{t}^{*}=\nu^{*} Y_{A t}^{*} & \nu^{*}>0
\end{array}
$$

Diferentemente de Matsuyama (1992), os parâmetros de velocidade de acumulação de produtividade são diferentes entre as economias, $\delta \neq \delta^{*}$ e $\nu \neq \nu^{*}$, refletindo a ausência de completo spillovers.

\subsubsection{Equilíbrio no mercado de trabalho}

Utilizando as equações 16 e 18, pode-se obter as à seguinte condição de equilíbrio no mercado de trabalho no resto do mundo:

$$
A_{t}^{*} g^{\prime}\left(l_{A t}^{*}=p_{t} I_{t}^{*} f^{\prime}\left(l_{I t}^{*}\right)\right.
$$

\section{DETERMINAÇÃO DO NÍVEL DE EMPREGO}

Considerando que não há barreiras comercias e nenhum tipo de distorção de preços, sob especialização incompleta, o emprego nos setores industrial e agrícola é determinado a partir das condições de equilíbrio no mercado de trabalho na economia doméstica e no resto do mundo.

Das equações 12 e 22 tem-se:

$$
\begin{aligned}
& \frac{f^{\prime}\left(l_{I t}\right)}{g^{\prime}\left(l_{A t}\right)}=\frac{A_{t}}{p_{t} I_{t}} \\
& \frac{f^{\prime}\left(l_{i t}^{*}\right)}{g^{\prime}\left(l_{A t}^{*}\right)}=\frac{A_{t}^{*}}{P_{t} I_{t}^{*}}
\end{aligned}
$$

Fazendo a razão entre essas duas equações, tem-se:

$$
\frac{f^{\prime}\left(l_{I t}\right)}{g^{\prime}\left(l_{A t}\right)}=\frac{A_{t} / I_{t}}{A_{t}^{*} / I_{t}^{*}} \frac{f^{\prime}\left(l_{I t}^{*}\right)}{g^{\prime}\left(l_{A t}^{*}\right)}
$$

Os setores agrícola e industrial competem por mão-de-obra de modo que uma elevação da produtividade agrícola desloca trabalhadores para este setor, reduzindo conseqüentemente o emprego e o nível de produção na indústria. Como os ganhos de produtividade em ambos os setores ocorrem devido a um processo de learning-by-doing existe uma relação negativa entre a produtividade agrícola e nível de emprego industrial, ou seja:

$$
l_{I t}^{*}=h\left(A_{t}^{*}\right) \quad h^{\prime}<0
$$

A partir das equações 23 e 24 pode-se estabelecer uma relação entre vantagens comparativas e nível de emprego. Em 23 a relação $f^{\prime}\left(l_{I l}\right) / g^{\prime}\left(l_{A t}\right)$ é decrescente em $l_{I t}$. Considerando-se um período inicial, $t=0$, se o setor agrícola da economia doméstica for relativamente mais produtivo que o do resto do mundo, $\left(A_{0} / I_{0}>A_{0}^{*} / I_{0}^{*}\right)$, ou seja, se a economia doméstica tem vantagens comparativas na agricultura, haverà um deslocamento da mão-de-obra do setor industrial para o setor agrícola na economia doméstica de modo que $l_{A 0}>l_{A 0}^{*}$. Por outro lado, se a economia doméstica tem vantagens comparativas na indústria, $A_{0} / I_{0}<A_{0}^{*} / I_{0}^{*}$, a participação do emprego industrial serà maior na economia doméstica que no resto do mundo, $l_{I 0}>l_{I 0}^{*}$. 


\subsection{Dinâmica do Emprego e do Produto na Economia Doméstica}

Considerando-se a participação da mão-de-obra na indústria e na agricultura da economia do resto do mundo como constante, substituindo 7 em 23, aplicando logaritmo e diferenciando 23 em relação ao tempo, obtém-se:

$$
\left[\frac{f^{\prime \prime}\left(j\left(l_{A t}\right)\right)}{f^{\prime}\left(j\left(l_{A t}\right)\right)} j^{\prime}\left(l_{A t}\right)-\frac{g^{\prime \prime}\left(l_{A t}\right)}{g^{\prime}\left(l_{A t}\right)}\right] \dot{l}_{A t}=\left[\frac{\dot{A}_{t}}{A_{t}}-\frac{\dot{A}_{t}^{*}}{A_{t}^{*}}\right]-\left[\frac{\dot{I}_{t}}{I_{t}}-\frac{\dot{I}_{t}^{*}}{I_{t}^{*}}\right]
$$

A equação 25 mostra a evolução do emprego na agricultura da economia doméstica ao longo do tempo. Se o diferencial de crescimento da produtividade agrícola na economia doméstica em relação ao resto do mundo for superior ao diferencial da evolução da produtividade na indústria, a participação do emprego no setor agrícola da economia doméstica crescerà, uma vez que o termo entre colchetes do lado esquerdo da equação é positivo.

A evolução do emprego também pode ser relacionada com os parâmetros de produtividade $\nu, \nu^{*}, \delta \mathrm{e}$ $\delta^{*}$. Substituindo 4 e 6 em 8 e 9 , respectivamente, obtém-se as taxas de crescimento das produtividades nos setores agrícola e industrial da economia doméstica, as quais são dadas, respectivamente, por, $\dot{A}_{t} / A_{t}=\nu T g\left(l_{A t}\right)$ e $\dot{I}_{t} / I_{t}=\delta K f\left(l_{I t}\right)$. As taxas de crescimento das produtividades no resto do mundo são obtidas substituindo-se 16 e 18 em 20 e 21, de onde se obtém, $\dot{A}_{t}^{*} / A_{t}^{*}=\nu^{*} T^{*} g\left(l_{A t}^{*}\right)$ e $\dot{I}_{t}^{*} / I_{t}^{*}=\delta^{*} K^{*} f\left(l_{I t}^{*}\right)$. Desse modo, a Equação 25 pode ser representada, alternativamente, como:

$$
\left[\frac{f^{\prime \prime}\left(j\left(l_{A t}\right)\right)}{f^{\prime}\left(j\left(l_{A t}\right)\right)} j^{\prime}\left(l_{A t}\right)-\frac{g^{\prime \prime}\left(l_{A t}\right)}{g^{\prime}\left(l_{A t}\right)}\right] i_{A t}=\left\{\left[\nu T g\left(l_{A t}\right)-\nu^{*} T^{*} g\left(l_{A t}^{*}\right)\right]-\left[\delta K f\left(l_{I t}\right)-\delta^{*} K^{*} f\left(l_{A t}^{*}\right)\right]\right\}
$$

A expressão 26, acima, fornece a dinâmica do emprego na agricultura da economia doméstica. Uma vez que a expressão dentro do colchete do lado esquerdo da igualdade é positiva, o grau de especialização de cada economia estarà condicionado aos sinais dos termos dentro dos colchetes no lado direito da desigualdade. Desse modo, o padrão de especialização da economia serà determinado pelas vantagens comparativas iniciais, pelos parâmetros de aprendizagem nos dois segmentos produtivos e pelas dotações de terra e capital, em cada economia.

Três situações podem ser descritas de modo a gerar assintoticamente especialização completa na agricultura, por parte da economia doméstica. A primeira ocorre quando a expressão dentro do primeiro colchete do lado direito da igualdade em 26 é positiva, $\frac{g\left(l_{A t}\right)}{g\left(l_{A t}^{*}\right)}>\frac{v^{*} T^{*}}{v T}$, e a segunda é negativa, $\frac{f\left(l_{A t}^{*}\right)}{f\left(l_{A t}\right)}>$ $\frac{\delta K}{\delta^{*} K^{*}}$. Nesse caso tem-se uma vantagem comparativa inicial na agricultura da economia doméstica em relação ao resto do mundo, $A / I_{0}>A^{*} / I_{0}^{*}$. Esse padrão de vantagem comparativa levaria a um processo mais acelerado de aprendizado nesse segmento $v>v^{*}$, e mais lento em relação à indústria, $\delta<\delta^{*}$; a uma maior utilização do fator terra, que é específico da agricultura, $T>T^{*}$, e a uma menor utilização do fator específico da indústria, $K<K^{*}$.

A segunda situação ocorre quando $\frac{g\left(l_{A t}\right)}{g\left(l_{A t}^{*}\right)}>\frac{v^{*} T^{*}}{v T}$ se mantêm, mas $\frac{f\left(l_{A t}^{*}\right)}{f\left(l_{A t}\right)}<\frac{\delta K}{\delta^{*} K^{*}}$. Nesta situação, não é necessàrio que o processo de aprendizado na indústria da economia doméstica seja mais lento quando comparado a do resto do mundo, $\delta<\delta^{*}$, e nem que a utilização do fator específico da indústria seja menos intensiva, $K<K^{*}$. O importante é que o termo positivo dentro do primeiro colchete seja maior que o do segundo colchete de modo que o resultado líquido ainda seja positivo. Deste modo, a única diferença em relação ao caso anterior é que o processo de especialização completa da economia doméstica em direção à agricultura ocorrerà de forma mais lenta.

Finalmente, a última possibilidade é considerar uma inversão de sinal dos termos dentro dos colchetes, em relação a situação anterior, ou seja, se $\frac{f\left(l_{A t}^{*}\right)}{f\left(l_{A t}\right)}>\frac{\delta K}{\delta^{*} K^{*}}$ e $\frac{g\left(l_{A t}\right)}{g\left(l_{A t}^{*}\right)}<\frac{v^{*} T^{*}}{v T}$. Neste contexto, a velocidade de aprendizado na indústria do resto do mundo, $\delta^{*}$, ponderado por $K^{*}$, mais que compensaria o fato dela também possuir uma alta taxa de aprendizado na agricultura $v^{*}$, ponderado da mesma forma pelo seu fator específico $T^{*}$, quando comparado aos parâmetros da economia doméstica. 
Neste sentido, o valor negativo do termo dentro da expressão do primeiro colchete do lado direito da igualdade, que poderia indicar uma tendência natural da economia doméstica em se especializar na indústria, seria neutralizada pela vocação na mesma direção e com maior intensidade, da economia do resto do mundo. $O$ efeito líquido final desse processo seria mais uma vez a tendência assintótica à especialização completa na agricultura pela economia doméstica. No entanto, assim como o caso anterior, esse movimento ocorreria de forma mais lenta quando comparado ao primeiro caso.

Como pode ser visto, os resultados apresentados na equação 26 estabelecem uma condição de equilíbrio no emprego na agricultura da economia doméstica mais ampla daquela obtida em Matsuyama (1992), onde os ganhos de produtividade nesse segmento são negligenciados. Essa hipótese corresponde considerar em $26 \nu=\nu^{*}=0$, de modo que as taxas de crescimento do emprego agrícola passam a ser subestimadas para economias que inicialmente apresentam vantagens comparativas na agricultura.

Um outro fato relevante é que em Matsuyama (1992), admite-se uma hipótese mais restritiva em que hà perfeito transbordamento do conhecimento entre os setores industriais da economia doméstica e do resto do mundo, tal que $\delta=\delta^{*}$. No modelo aqui apresentado, relaxa-se esse fato possibilitando uma situação mais geral em que $\nu \neq \nu^{*}$ e $\delta \neq \delta^{*}$. Essa suposição passa a ser crucial para alguns resultados adicionais, na medida em que ela abre espaço para a possibilidade de haver, por parte de uma determinada economia, uma tendência assintótica de especialização completa em um segmento distinto daquele na qual a economia apresente uma vantagem comparativa inicial.

Na medida em que $i_{A t}$ é influenciado pela magnitude dos parâmetros de $\nu, \nu^{*}, \delta$ e $\delta^{*}$, políticas que objetivem alterar seus valores podem tanto reforçar seu padrão potencial de especialização como reverter essa tendência inicial. Como visto anteriormente, nas três situações descritas anteriormente, o sinal de $i_{A t}$ é dado pela relação de grandeza existente entre os termos $\frac{g\left(l_{A t}\right)}{g\left(l_{A t}^{*}\right)}$ e $\frac{v^{*} T^{*}}{v T}$ assim como entre $\frac{f\left(l_{A t}^{*}\right)}{f\left(l_{A t}\right)}$ e $\frac{\delta K}{\delta^{*} K^{*}}$. Essas relações podem ter o sinal de desigualdade alterado caso haja mudanças importantes nas magnitudes dos parâmetros de aprendizado.

No primeiro caso, por exemplo, considerou-se a possibilidade de especialização completa pela economia doméstica na agricultura a partir da existência das condições em que $\frac{g\left(l_{A t}\right)}{g\left(l_{A t}^{*}\right)}>\frac{v^{*} T^{*}}{v T} \mathrm{e} \frac{f\left(l_{A t}^{*}\right)}{f\left(l_{A t}\right)}>$ $\frac{\delta K}{\delta^{*} K^{*}}$. Esta situação seria atendida, entre outras suposições, considerando que a economia doméstica tenha vantagem comparativa inicial na agricultura e que $v>v^{*}$ e $\delta<\delta^{*}$.

No entanto, se alguma política for capaz de alterar $v^{*}$ e $\delta$, de modo que eles passem a ser superior a $v$ e $\delta^{*}$ respectivamente, a direção da desigualdade pode ser alterada, implicando que mesmo que a economia doméstica tenha vantagem comparativa inicial na agricultura, ela possa assintoticamente se especializar completamente na indústria e o inverso ocorrendo com o resto do mundo.

Nesse sentido, este modelo permite a possibilidade de reversão de especialização a partir do padrão inicial de vantagens comparativas, o que não é possível em Matsuyama (1992). Se $v=v^{*}=0$ e $\delta=\delta^{*}$, na equação 26, obtêm-se o mesmo resultado de Matsuyama de modo que somente as vantagens comparativas iniciais é que importam para determinar o nível de especialização em cada economia.

Considerando que o produto total é a soma do produto de cada setor, a partir das equações que representam as tecnologias de produção na indústria (1) e na agricultura (5), a oferta total de mão de obra (2) e a evolução da produtividade industrial (8) e agrícola (9), obtém-se a taxa de crescimento do produto na economia doméstica, a qual é dada por:

$$
\frac{\dot{Y}}{Y}=\delta F\left(1-L_{A T}, K\right)+\nu G\left(L_{A T}, T\right)+\left(\frac{G_{L_{A T}}}{G\left(L_{A T}, T\right)}-\frac{F_{L_{I T}}}{F\left(L_{I T}, K\right)}\right) \cdot \dot{L}_{A T}
$$

onde $G_{L_{A T}}$ e $F_{L_{I T}}$, representam, respectivamente, as produtividades marginais da mão-de-obra empregada nos setores agrícola e industrial.

Como pode ser visto pela Equação 27, a taxa de crescimento do produto da economia doméstica està condicionada também aos valores dos parâmetros $\delta$ e $\nu$, os quais determinam à velocidade de 
acumulação de conhecimento nos setores industrial e agrícola, respectivamente. Desse modo, se a taxa de acumulação do conhecimento na agricultura for muito elevada em relação à indústria à economia poderà ter um grande setor agrícola e crescer a taxas elevadas.

Ademais, das equações 23 a 27, pode-se verificar que se inicialmente as produtividades relativas da agricultura nas duas economias forem iguais, os níveis de emprego na agricultura e indústria também serão. Um aumento da produtividade relativa da agricultura na economia doméstica, provocarà um deslocamento da mão-de-obra para este setor, de modo que, $l_{A t}>l_{A}^{*}$.

Deste modo, diferentemente do resultado obtido em Matsuyama (1992), que considerava a existência uma relação negativa entre a produtividade agrícola e crescimento econômico em economias abertas sem learnig-by-doing na agricultura, esse modelo aponta para o fato de que a introdução de um processo de aprendizado também nesse setor levaria a um deslocamento da mão-de-obra para este segmento ocasionando um aumento de sua produção o que provocaria um impacto produtividade na renda da economia como um todo.

\section{RELAÇÃO ENTRE PRODUTIVIDADE AGRÍCOLA E BEM-ESTAR}

Um outro ponto relevante do modelo é analisar os impactos da produtividade agrícola sobre bemestar da economia. Considerando-se a renda nacional da economia doméstica, $R_{t}$, como sendo a soma dos valores dos bens produzidos nos setores agrícola e industrial, ou seja:

$$
R_{t}=A_{t} g\left(l_{A t}\right)+p_{I} I_{t} f\left(l_{I t}\right)
$$

O dispêndio nacional per capita $\left(E_{t} / L\right)$ será dado por:

$$
\frac{E_{t}}{L}=c_{A t}+p_{t} c_{I T}
$$

A função utilidade instantânea do agente representativo na economia doméstica é dada pela Equação 14. Fazendo $\sigma=1 \mathrm{em} \mathrm{14}$, o problema do indivíduo em cada período de tempo serà maximizar a seguinte função utilidade:

$$
M a x U=\int_{0}^{\infty}\left[\beta \log \left(c_{A t}-\gamma\right)+\log \left(c_{I t}\right)\right] e^{-\rho t} d t
$$

Sujeito a $c_{A t}+p_{t} c_{I t} \leq R_{t} / L$.

Do problema 30 pode-se obter a demanda per capita por bens agrícolas em função dos preços relativos e da demanda por bens industrializados (per capita), como sendo:

$$
c_{A t}=\gamma+\beta p_{t} c_{I t}
$$

Substituindo 31 em 30 tem-se:

$$
U=\int_{0}^{\infty}\left[\beta \log \left(\beta p_{t} c_{I t}\right)+\log \left(c_{I t}\right)\right] e^{-\rho t} d t
$$

De 29 tem-se:

$$
c_{A t}=E_{t} / L-p_{t} c_{I t}
$$

Substituindo 31 em 33 pode-se obter a demanda per capita por bens industrializados:

$$
c_{I t}=\frac{\left(E_{t} / L\right)-\gamma}{(1+\beta) p_{t}}
$$

Substituindo 34 em 32, obtém-se a função utilidade indireta do agente representativo, 


$$
h=(1+\beta) \int_{0}^{\infty} \log \left(\frac{E_{t}}{L}-\gamma\right) e^{-\rho t} d t+\Psi
$$

onde $\Psi=-\int_{0}^{\infty} \log \left(p_{t}\right) e^{-\rho t} d t+\int_{0}^{\infty}[\beta \log \beta+(1+\beta) \log (1+\beta)] e^{-\rho t} d t$.

A partir da equação 35 pode-se analisar o bem estar da economia doméstica relacionando-o com seu nível de renda agregada, $R_{t}$, considerando duas situações: na primeira, assume-se que não existe mercado de capitais, ou seja, não hà possibilidade da economia doméstica emprestar ou adquirir empréstimos do resto do mundo, de modo que $R_{t}=E_{t}$ em cada período de tempo. Assim,

$$
h_{1}=(1+\beta) \int_{0}^{\infty} \log \left(\frac{R_{t}}{L}-\gamma\right) e^{-\rho t} d t+\Psi
$$

Na segunda situação considera-se a existência de um perfeito mercado de capitais, de modo que a economia doméstica pode emprestar e obter empréstimos a uma taxa de juros constante $(r)$ a qual é igual a taxa de desconto $(\rho)$. Desse modo, a economia doméstica gasta uma quantidade constante em cada período de tempo, dada por:

$$
\frac{E_{t}}{\rho}=\int_{0}^{\infty} R_{t} e^{-\rho t} d t
$$

onde o termo do lado esquerdo corresponde ao valor presente da despesa descontada a uma taxa $r$.

Substituindo 37 em 35:

$$
h_{2}=(1+\beta) \rho^{-1} \log \left[\rho \int_{0}^{\infty} \frac{R_{t}}{L} e^{-\rho t} d t-\gamma\right]+\Psi
$$

A renda do resto do mundo é dada por:

$$
R_{t}^{*}=A_{t}^{*} g\left(l_{A t}^{*}\right)+p_{I} I_{t}^{*} f\left(l_{I t}^{*}\right)
$$

Multiplicando e dividindo o segundo termo do lado direito da Equação 22 por $f\left(l_{I t}^{*}\right)$, e substituindo em 39 obtém-se:

$$
R_{t}^{*}=A_{t}^{*}\left[g\left(l_{A}^{*}\right)+g\left(l_{A}^{*}\right) \frac{f\left(l_{I}^{*}\right)}{f^{\prime}\left(l_{I}^{*}\right)}\right]
$$

Se a produtividade da agricultura na economia doméstica for igual a do resto do mundo, a participação da mão-de-obra empregada nos setores agrícola e industrial serão iguais em todas as economias, de modo que a renda da economia doméstica e do resto do mundo serão iguais, $R_{t}=R_{t}^{*}$, e constantes ao longo do tempo.

Fazendo $R_{t}=R_{t}^{*}=R$ em 40 e substituindo o resultado em 38 , tem-se que

$$
h_{2}=(1+\beta) \rho^{-1} \log \left(\frac{R}{L}-\gamma\right)+\Psi
$$

Da Equação 41 verifica-se que se não hà vantagens comparativas entre as economias, o nível de bem-estar da economia doméstica independe da existência de mercados de capitais internacionais, isto é, $h_{1}=h_{2}=(1+\beta) \rho^{-1} \log \left(\frac{R_{t}^{*}}{L}-\gamma\right)+\Psi$. Isso decorre do fato de não haver deslocamento de mão-de-obra entre os setores da economia e conseqüentemente não haver alteração da renda ao longo do tempo. Desde que a medida de bem-estar depende do nível de renda da economia, esta também permanecerà inalterada.

Os resultados obtidos por Matsuyama (1992) indicam que a renda de uma economia e, conseqüentemente, o seu bem-estar crescerà se a economia se industrializar. Porém, o modelo aqui apresentado 
considera a existência de learning-by-doing em ambos os setores chegando a um resultado mais geral em que se. Se $A \neq A^{*}$, a renda nacional e o nível de bem-estar crescerão independentemente do setor em que a economia apresentar vantagens comparativas. 0 crescimento da renda ocorre devido ao deslocamento da mão-de-obra entre os setores, de modo que se a produtividade agrícola da economia doméstica é maior que no resto do mundo, isto é, $A>A^{*}$, a mão-de-obra se deslocarà do setor industrial para a agricultura. Se, alternativamente, $A<A^{*}$, tem-se que $i_{I}>0$ e $i_{A}<0$. No entanto, os níveis de renda e bem estar crescerão a taxas mais elevadas se a economia se especializar no setor em que apresenta vantagens comparativas iniciais.

Por fim, como lembrado em Matsuyama (1992), mesmo que a agricultura não tenha dinâmica ou que esta dinâmica seja baixa em relação à indústria não se deve usar esse argumento negligenciar a agricultura de um país em prol da industrialização. Considerando que a produtividade agrícola de uma economia seja suficientemente alta, seu nível de renda e bem-estar poderão ser mais elevados que no resto do mundo. Assim, um país que se especializa na agricultura poderà crescer mais lentamente $\mathrm{e}$ mesmo assim não apresentar um padrão de vida mais baixo.

\section{CONCLUSÕES}

Esse artigo utiliza um modelo de crescimento endógeno em um ambiente de livre comércio para analisar as implicações da incorporação dos avanços tecnológicos obtidos através do processo de learningby-doing sobre o nível de emprego e bem-estar da economia. A estrutura teórica està baseada no modelo proposto por Matsuyama (1992). O modelo originalmente proposto difere do modelo aqui apresentado em relação a duas suposições bàsicas. Primeiro considera-se que a dinâmica da economia està condicionada a evolução da produtividade nos setores agrícola e industrial. Adicionalmente, supõe-se que não hà completo spillovers entre as economias.

Com base nos resultados obtidos verificou-se que as conclusões obtidas por Matsuyama (1992) representam um caso particular de conclusões mais gerais, onde a especialização da economia no setor agrícola não necessariamente implica em perda de bem-estar no curto e no longo prazo.

Em relação ao nível e evolução da mão-de-obra entre os setores da economia, verificou-se que se inicialmente nas duas economias a produtividade da agricultura em relação à indústria são iguais, os níveis de emprego nesses dois setores também serão. Um aumento na produtividade relativa da agricultura na economia doméstica provocarà um deslocamento da mão-de-obra para este setor.

Em termos de trajetória do emprego agrícola, tem-se que se a economia doméstica tem vantagem comparativa na agricultura em um período inicial, a taxa de crescimento do emprego nesse setor serà positiva. Se hà um aumento da taxa de crescimento da produtividade agrícola na economia doméstica, a trajetória de crescimento do emprego agrícola dessa economia se deslocarà para cima. No entanto, quando se negligencia os ganhos de produtividade na agricultura, as taxas de crescimento do emprego agrícola para economias que inicialmente apresentam vantagens comparativas na agricultura são subestimadas.

A velocidade de crescimento do emprego também pode ser influenciada pela magnitude dos parâmetros de produtividade da economia doméstica e do resto do mundo. Se este último ampliar sua produtividade agrícola a economia doméstica perde suas vantagens comparativas tornando-se menos especializada. A especialização completa das economias em um dos setores dependerà, no entanto, das propriedades das funções de produção na origem; de modo que, no longo prazo, a economia doméstica poderà se especializar na agricultura caso, inicialmente, ela apresente vantagens comparativas nesse setor.

Matsuyama (1992) verificou que em economias abertas, hà uma relação negativa entre produtividade agrícola e crescimento econômico. Porém quando se introduz um processo de learning-by-doing também na agricultura isso não necessariamente ocorre. 0 crescimento da economia dependerà agora da relação entre os parâmetros que determinam as taxas de acumulação de conhecimento nos dois setores. Se a 
taxa de acumulação de conhecimento na agricultura for maior que na indústria, a economia poderà ter um grande setor agrícola e mesmo assim crescer a elevadas taxas.

Se não há vantagens comparativas entre as economias, a mão-de-obra não se deslocarà entre os setores, de modo que não ocorre nenhuma alteração no nível de renda ao longo do tempo. Como a medida de bem-estar depende do nível de renda da economia, esta também permanecerà inalterada, e independente da existência de mercados de capitais internacionais.

Os resultados obtidos por Matsuyama (1992) indicam que a renda de uma economia e, conseqüentemente, o seu bem-estar crescerà se a economia se industrializar. Porém, o modelo aqui apresentado mostra que a renda nacional e o nível de bem-estar crescerão independentemente do setor em que a economia apresenta vantagens comparativas. No entanto, os níveis de renda e bem estar crescerão a taxas mais elevadas se a economia se especializar no setor em que apresenta vantagens comparativas iniciais. Assim, um país que se especializa na agricultura poderà crescer mais lentamente e mesmo assim apresentar um padrão de vida mais elevado.

\section{Referências Bibliográficas}

Barros, A. M. (1999). Capital, Produtividade e Crescimento da Agricultura: o Brasil de 1970 e 1995. PhD thesis, Escola Superior de Agricultura "Luiz de Queiroz", Universidade de São Paulo.

Battese, G. E. \& Rao, D. P. (2001). Productivity potential and technical efficiency levels of firms in different regions using a stochastic frontier metaproduction function model. Technical Report 6, CEPA.

Battese, G. E., Rao, D. P., \& Walujadi, D. (2001). Technical efficiency and productivity of garment firms in different regions in indonesia: A stochastic frontier analysis using a time- varying inefficiency model and a metaproduction function. Technical Report 7, CEPA.

Ben-David, D. (1994). Convergence clubs and diverging economies. Technical Report 922, CEPR. Discussion Papers.

Bureau, J., Faere, R., \& Grosskopf, S. (1995). A comparison of three nonparametric measures of productivity growth in european and united states agriculture. Journal of Agricultural Economics, 46:309-326.

Easterly, W. (1991). Economic stagnation, fixed factors, and policy thresholds. Journal of Monetary Economics, 33:525-557.

Fulginiti, L. E. \& Perrin, R. K. (1993). Prices and productivity in agriculture. Review of Economics and Statistics, 84:471-482.

Grossman, G. M. \& Helpman, E. (1989). Product development and international trade. Journal of Political Economy, 97(6):1261-83.

Grossman, G. M. \& Helpman, E. (1990). Comparative advantage and long-run growth. American Economic Review, 80(4):796-815.

Grossman, G. M. \& Helpman, E. (1991a). Innovation and Growth in thew Global Economy. Mass: MIT Press, Cambridge.

Grossman, G. M. \& Helpman, E. (1991b). Quality ladders and product cycles. Quarterly Journal of Economics, 106:557-586.

Kawagoe, T., Hayami, Y., \& Ruttan, V. (1985). The intercountry agricultural production function and productivity differences among countries. Journal of Development economics, 19:113-132.

Krugman, P. R. (1990). Rethinking International Trade. Cambridge MIT Press, Cambridge. 
Lau, L. \& Yotopoulos, P. (1989). The meta-production function approach to technological change in world agriculture. Journal of Development Economics, 31:241-269.

Lucas, R. E. (1988). On the mechanics of economic development. Journal of Monetary Economics, 22:3-42.

Marinho, E. L. L. \& Carvalho, R. M. (2004). Comparações inter-regionais da produtividade da agricultura brasileira 1970-1995. Pesquisa e Planejamento Econômico, 34(1).

Matsuyama, K. (1992). Agricultural productivity, comparative advantage, and economic growth. Journal of Economic Theory, 58:317-334.

Prasadasa, D. S. R. \& Coelli, T. J. (1998). Catch-up and convergence in global agricultural productivity 1980 - 1995. CEPA Working Papers, 4/98. University of New England. Australia.

Quad, D. T. (1997). Empiric for growth and distribution: Stratification, polarization, and convergence clubs. Journal of Economic Growth, 2.

Rebelo, S. (1992). Growth in open economies. Carnegie Rochester Conference Series on Public Polic, 36:5-46.

Rivera-Batiz, L. A. \& Romer, P. M. (1991a). Economic integration and endogenous growth. Quarterly Journal of Economics, 106:531-555.

Rivera-Batiz, L. A. \& Romer, P. M. (1991b). International trade with endogenous technological change. European Economic Review, 35:97-1001.

Romer, P. M. (1990). Endogenous technological change. Journal of Political Economy, 98:S71-S102.

Sharif, M. (1986). The concept and measurement of subsistence: a survey of the literature. World Development, 14:555-577.

Steger, T. M. (2000). Economic growth with subsistence consumption. Journal of Development Economies, 62:343-361.

Stokey, N. L. (1991). The volume and composition of trade between rich and poor countries. Review of Economic Studies, 58:701-717.

Vicente, J. R., Anefalos, L. C., \& Caser, D. V. (2001). Produtividade agrícola no brasil, 1970-1995. Agricultura em São Paulo. São Paulo, 48:35-55.

Wong, K. (1995). International Trade in Goods and Factor Mobility. MIT PRESS, Cambridge, Massachusetts. London, England.

World, B. (1990). World Development Report: Poverty. Oxford Univ. Press, New York.

Young, A. (1991). Learning by doing and the dynamic effects of international trade. Quarterly Journal of Economics, 106:369-405. 